\title{
Optimisation des paramètres de dépôt de a-Si:H dans la technique Hg-photo-CVD
}

\author{
Boko AKA \\ Laboratoire de Physique Appliquée, Ecole Normale Supérieure d'Abidjan, \\ 22BP 1561 Abidjan 22 (Côte d'Ivoire)
}

(Reçu le II Février 2005, accepté le 23 juin 2005)

*Correspondance et tirés à part, e-mail : a.bokom@yahoo.fr

\section{Résumé}

La technique $\mathrm{Hg}$-photo-CVD, reconnue pour son usage dans l'élaboration en couches minces de matériaux semi-conducteurs et diélectriques, connaît un regain d'intérêt au vu de leurs nombreuses applications en microélectronique.

II est établi que les propriétés physico-chimiques et optoélectroniques du matériau déposé, en l'occurrence le silicium amorphe hydrogéné (a-Si:H), sont fonction des conditions de préparation, notamment la température du substrat, la pression des gaz réactifs, leur débit, l'intensité lumineuse, la température du milieu réactionnel, la température du mercure et la température du recuit post-dépôt.

Nous dégageons dans cet article qui est une revue de nos travaux en la matière, les conditions d'optimisation des principaux paramètres du dépôt: Température du mercure $\mathrm{T}_{\mathrm{Hg}}$ : environ $50^{\circ} \mathrm{C}$ - Température du substrat $\mathrm{T}_{\mathrm{s}}: 250$ à $300^{\circ} \mathrm{C}$ - Pression du gaz réactif : autour de 1 Torr - Débit du gaz réactif : 1 à $5 \mathrm{sccm}\left(0 u \mathrm{~cm}^{3} / \mathrm{s}\right)$ - L'intensité lumineuse nominale : 10 à $20 \mathrm{~mW} / \mathrm{cm}^{2}$, température du recuit post- dépôt $200-300{ }^{\circ} \mathrm{C}$ pendant 30 minutes.

A $T_{s}=280^{\circ} \mathrm{C}$, la photosensibilité est optimale avec une valeur de plus de $10^{3}$. Dans les conditions de travail optimales proposées, la conversion du gaz réactif en silicium est rationalisée et la quantité élevée d'hydrogène formé sert, pour des raisons de sécurité, de gaz vecteur pour l'évacuation du mélange gazeux.

Mots-clés : silicium amorphe hydrogéné (a-Si:H), Hg-photo-CVD, optimisation, photosensibilité. 


\section{Abstract}

\section{Optimizing deposition parameters of a-Si:H in Hg-photo-CVD technology}

The Hg-photo-CVD technique, known for its use in developing thin film of semiconductor materials and dielectrics has been a resurgence of interest in view of their many applications in microelectronics. It is established that the physico-chemical and optoelectronic material filed in this case the hydrogenated amorphous silicon (a$\mathrm{Si}: \mathrm{H}$ ) are a function of preparation conditions, including substrate temperature, the pressure of reactive gases, their speed, light intensity, temperature of reaction medium, temperature of the mercury and temperature of the post-deposition annealing.

We highlight in this article which is a review of our work in this area, the conditions for optimization of key parameters of deposition : Mercury Temperature $T_{H G}$ : about 50 ${ }^{\circ} \mathrm{C}$ - substrate temperature Ts : 250 to $300^{\circ} \mathrm{C}$ - Reactive gas pressure : around 1 Torr - gas flow reagent : 1 to $5 \mathrm{sccm}$ (or $\mathrm{cm}^{3} / \mathrm{s}$ ) - The nominal light intensity : 10 to 20 $\mathrm{mW} / \mathrm{cm}^{2}$, temperature of post-deposition annealing: about $300^{\circ} \mathrm{C}$ for 30 minutes.

At Ts $=280^{\circ} \mathrm{C}$, the photosensitivity is optimized to a value of more than $10^{3}$. In the optimal working conditions proposed, conversion of the reactive gas in silicon is streamlined and the large amount of hydrogen formed, for security reasons, become as the carrier gas for the evacuation of the gas mixture.

Keywords : Hydrogenated amorphous silicon (a-Si:H), Hg-photo-CVD, optimization, photosensitivity.

\section{Introduction}

Parmi les nombreuses techniques d'élaboration de silicium amorphe hydrogéné (a-Si:H) en couches minces (Pyrolyse CVD, Sputtering, Plasma, photo-CVD, ...), les techniques photo-assistées ou simplement Photo-CVD constituent une voie prometteuse qui permet le dépôt de a-Si:H à partir de gaz réactif en général $\mathrm{SiH}_{4}$, $\mathrm{Si}_{2} \mathrm{H}_{6}$ ou même $\mathrm{Si}_{3} \mathrm{H}_{8 .}$, soit pur soit en dilution le plus souvent avec l'azote, l'hélium ou même l'hydrogène.

On sait aussi que les propriétés physico-chimiques du matériau obtenu sont fonction des conditions de préparation (notamment le débit des gaz réactifs, la température du 
milieu réactionnel, la température du substrat, l'intensité lumineuse, et la température du mercure dans le cas de la méthode Hg-photo-(VD).

Il est établi sans équivoque que les produits gazeux issus de la décomposition de $\mathrm{SiH}_{4}$ sont dans l'ordre d'importance décroissante $\mathrm{H}_{2}, \mathrm{Si}_{2} \mathrm{H}_{6}, \mathrm{Si}_{3} \mathrm{H}_{8}$. On observe aussi un dépôt solide sur les parois du réacteur, dépôt qui peut être sélectivement dirigé en grande partie vers un substrat chauffé.

Les techniques photo-assistées rendent possibles des dépôts à des températures relativement basses (comparées par exemple à la pyrolyse (VD), à la fois dans le milieu réactionnel (même jusqu'à $25^{\circ} \mathrm{C}$ ) que pour le chauffage sélectif du susbrat (de 100 à $600^{\circ} \mathrm{C}$ ). La décomposition photosensibilisée au mercure du silane par une lampe à vapeur de mercure sous faible pression (Hg-photo-CVD ) fait l'objet de ce travail.

Cette méthode, utilisée depuis le début de ce siècle pour des études de chimie, a trouvé dans les années 1980 [1-4] son intérêt dans la déposition de films minces dès lors qu'il a été nécessaire de rechercher de nouveaux procédés d'élaboration de silicium à basse température $\left(\mathrm{T}<400^{\circ} \mathrm{C}\right)$ et à bon marché.

La lampe à vapeur de mercure sous faible pression émet deux radiations de résonance à des longueurs d'onde de 184,9 nm (soit $185 \mathrm{~nm}$ ) et 253,7 nm (soit $254 \mathrm{~nm}$ ). Celle émise à 184,9 nm est totalement absorbée par les parois du tube de la lampe et du réacteur, les deux étant en quartz.

Le monosilane $\left(\mathrm{SiH}_{4}\right)$ présente une très faible absorption pour les longueurs d'onde supérieures à $165 \mathrm{~nm}$. Par conséquent, la lumière émise à 253,7 nm n'a aucun effet sur les molécules de $\mathrm{SiH}_{4}$. La présence de quelques gouttes de mercure, dont la tension de vapeur à $25^{\circ} \mathrm{C}$ est de l'ordre de $2.10^{-3}$ Torr, suffit à induire la décomposition de $\mathrm{SiH}_{4}$.

La radiation émise à 253,7 $\mathrm{nm}$ est absorbée par résonance par les atomes de la vapeur de mercure présents dans le réacteur qui passent de ce fait de l'état fondamental $\mathrm{Hg}\left({ }^{1} S_{0}\right)$ à l'état excité $\mathrm{Hg}\left({ }^{3 P_{1}}\right)$. Par collision, ces derniers transfèrent leur excès d'énergie aux molécules de $\mathrm{SiH}_{4}$ qui peuvent alors se décomposer.

Cet article passe en revue nos différents travaux en la matière, en essayant de dégager les conditions optimales d'exploitation du procédé $\mathrm{Hg}$-photo-CVD. 


\section{Méthodologie}

Pour l'étude fondamentale, une grande partie du travail a porté sur la cinétique de dépôt afin d'élucider le comportement en phase gazeuse et en surface des espèces réactionnelles conduisant à la croissance de la couche. Dans ce but, la composition du gaz a été mesurée en fonction du temps d'irradiation pour différentes pressions initiales en silane de même que la quantité de silicium déposée analysée par absorption atomique [5-10].

Le mélange gazeux résultant de la photolyse de $\mathrm{SiH}_{4}$ est constitué principalement de $\mathrm{H}_{2}$, $\mathrm{SiH}_{4}$ et de $\mathrm{SiH}_{4}$ non décomposé et quelque fois d'une infime quantité de dérivés supérieurs de $\mathrm{SiH}_{4}\left(\mathrm{Si}_{3} \mathrm{H}_{8}, \mathrm{Si}_{3} \mathrm{H}_{10}, \ldots\right)$. La technique d'analyse utilisée est basée sur la différence des points de liquéfaction des constituants gazeux de la réaction :

$$
\mathrm{TH}_{2}=-253^{\circ} \mathrm{C}: \mathrm{T}_{\mathrm{SiH} 4}=-112^{\circ} \mathrm{C}: \mathrm{T}_{\mathrm{si2H}}=-14^{\circ} \mathrm{C} \text {. }
$$

A l'arrêt de l'irradiation, le mélange gazeux est refroidi dans de l'azote liquide $\left(\mathrm{T}=-196^{\circ} \mathrm{C}\right)$. Dans ces conditions, tous les gaz sont liquéfiés sauf l'hydrogène moléculaire $\left(\mathrm{H}_{2}\right)$ dont on mesure alors la pression. Les molécules d'hydrogène étant chassées hors du réacteur, on laisse remonter la température tout en notant la pression due à $\mathrm{SiH}_{4}$ et ensuite la pression due à $\mathrm{SiH}_{4}$ et à $\mathrm{Si}_{2} \mathrm{H}_{6}$. Une courbe d'étalonnage, Pression $=\mathrm{f}$ (température), préalablement établie avec du silane pur $\left(\mathrm{SiH}_{4}\right)$, de l'hydrogène pur $\left(\mathrm{H}_{2}\right)$, et de mélange de titre connu de silane et d'hydrogène permet d'accéder aux pressions partielles réelles de chacun des principaux constituants du mélange gazeux.

La caractérisation des couches a considéré, d'une part, pour les propriétés structurales, en l'analyse des échantillons par des techniques de spectroscopie infrarouge (IR), analyse par détection des reculs élastiques (ERDA), spectroscopie de masse d'ions secondaires (SIMS), rétrodiffusion Rutherford (RBS) et microscopie électronique à balayage (SEM) et, d'autre part, en la mesure et au calcul des paramètres optiques et électroniques en fonction des conditions de préparation et du recuit. Dans ce travail, seules les mesures jugées pertinentes ont été présentées.

\section{Résultats et discussion}

On observe à la Figure $I$ que les pressions partielles atteignent un palier après quelques minutes d'irradiation, alors que la transmission que nous avions mesurée à $\lambda=253,7 \mathrm{~nm}$ montre que les parois sont encore transparentes. Par contre, la 
quantité de silicium déposée augmente sensiblement et continuellement avec le temps d'irradiation.
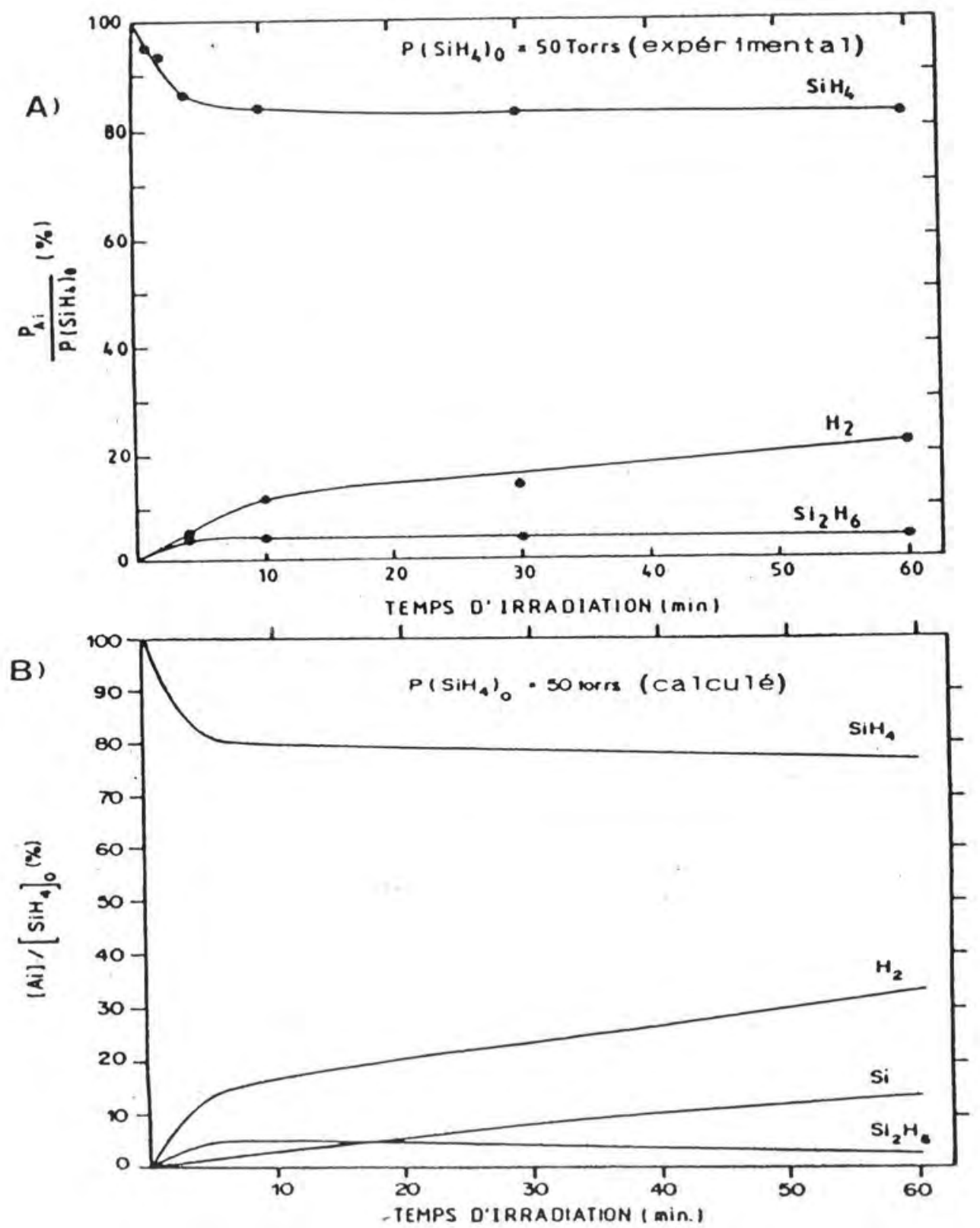

Figure 1 : Evolution des espèces du milieu réactionnel dans la technique Hg-photo-CVD pour une pression initiale de silane de 50 Torrs
A) Valeurs mesurées
B) valeurs simulées 


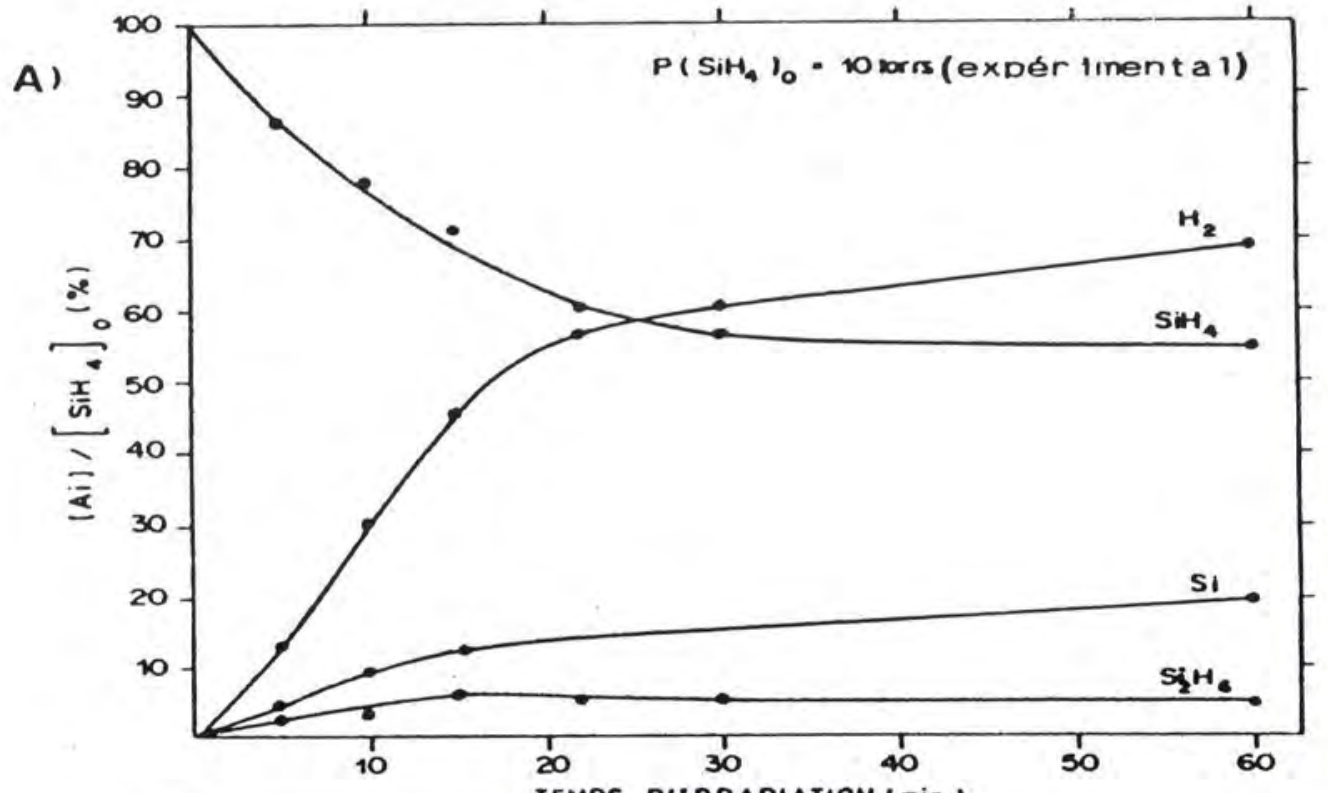

B)

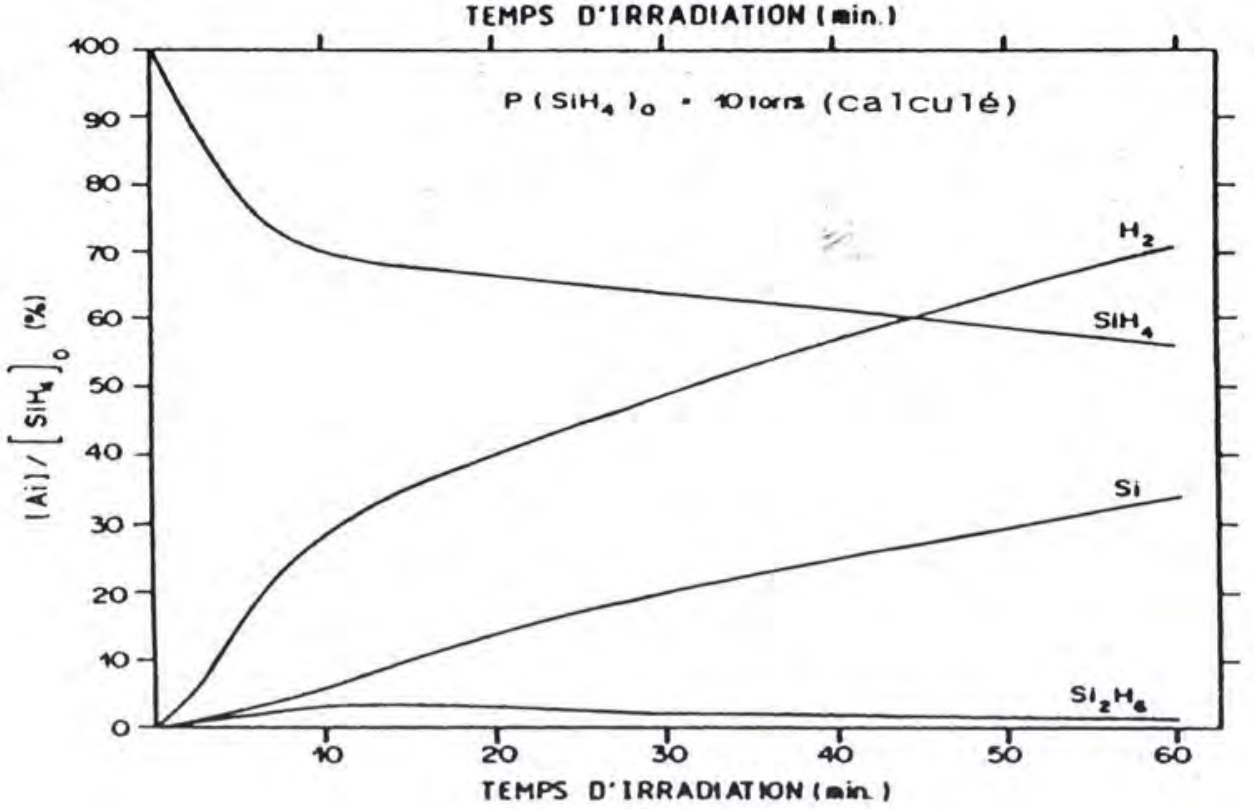

Figure 2 : Evolution des espèces du milieu réactionnel dans la technique Hg-photo-CVD pour une pression initiale de silane de 10 Torrs

$\begin{array}{ll}\text { A) Valeurs mesurées } & \text { B) valeurs simulées }\end{array}$ 
Nous avons mis en évidence que le palier atteint par le réactif et les produits gazeux issus de sa décomposition est un état photostationnaire conformément aux lois de l'équilibre chimique. Le palier n'est pas causé par la baisse de l'intensité lumineuse due au dépôt de silicium sur les parois.

La quantité de silane décomposée est limitée par le mécanisme chimique de la photolyse faisant intervenir des réactions élémentaires directes et inverses et de reformation des molécules de $\mathrm{SiH}_{4}$.

Un modèle chimique a été proposé, tenant compte des réactions en phase gazeuse et de l'absorption en surface des radicaux libres conduisant à la croissance de la couche de silicium amorphe a-Si.

En absorbant, par résonance, le rayonnement UV l'atome de mercure passe de l'état fondamental $\left(6^{1} S_{0}\right)$ à l'état excité singulet ${ }^{1} P_{1}$ ou triplet ${ }^{3} P_{1}$ selon le processus $[1-16]$ :

$\mathrm{Hg}\left({ }^{\left(\mathrm{S}_{0}\right)}+\mathrm{hV}(254 \mathrm{~nm})\right.$

Par collision sur l'atome de mercure excité, le silane se décompose principalement comme suit :

$\mathrm{Hg}^{*}\left({ }^{3} \mathrm{P}_{1}\right)+\mathrm{SiH}_{4}$

La réaction primaire peut être accompagnée par un ou plusieurs processus secondaires tels que :

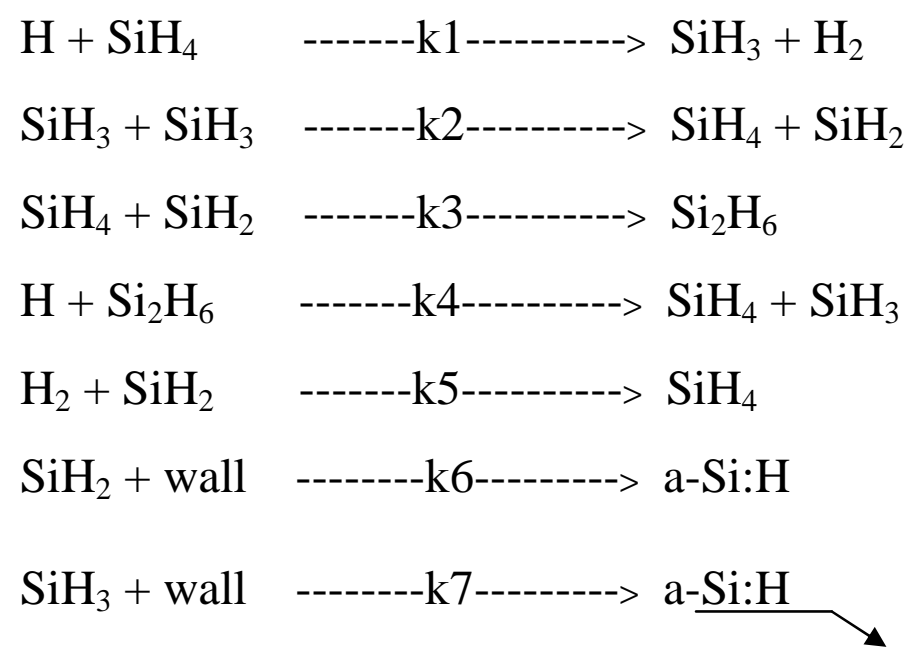

Schéma 1 : schéma du modèle cinétique simple proposé

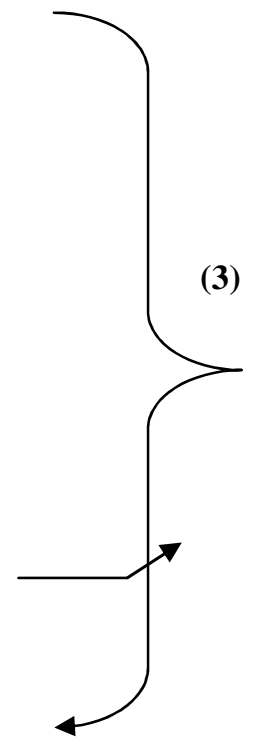


Le calcul cinétique du modèle par des méthodes numériques avec les constantes de vitesse appropriées permet d'interpréter avec satisfaction les résultats expérimentaux (Figures IB, $2 B$ et 4 ).

Il est intéressant de noter que par le même calcul, étendu sur un large domaine de pression, on prévoit un maximum d'efficacité dans la technique $\mathrm{Hg}$-photo-CVD en opérant à basse pression ( $\mathrm{P}<1$ Torr) (Figure 3), ce qui est en conformité avec les résultats expérimentaux (Figure 4).

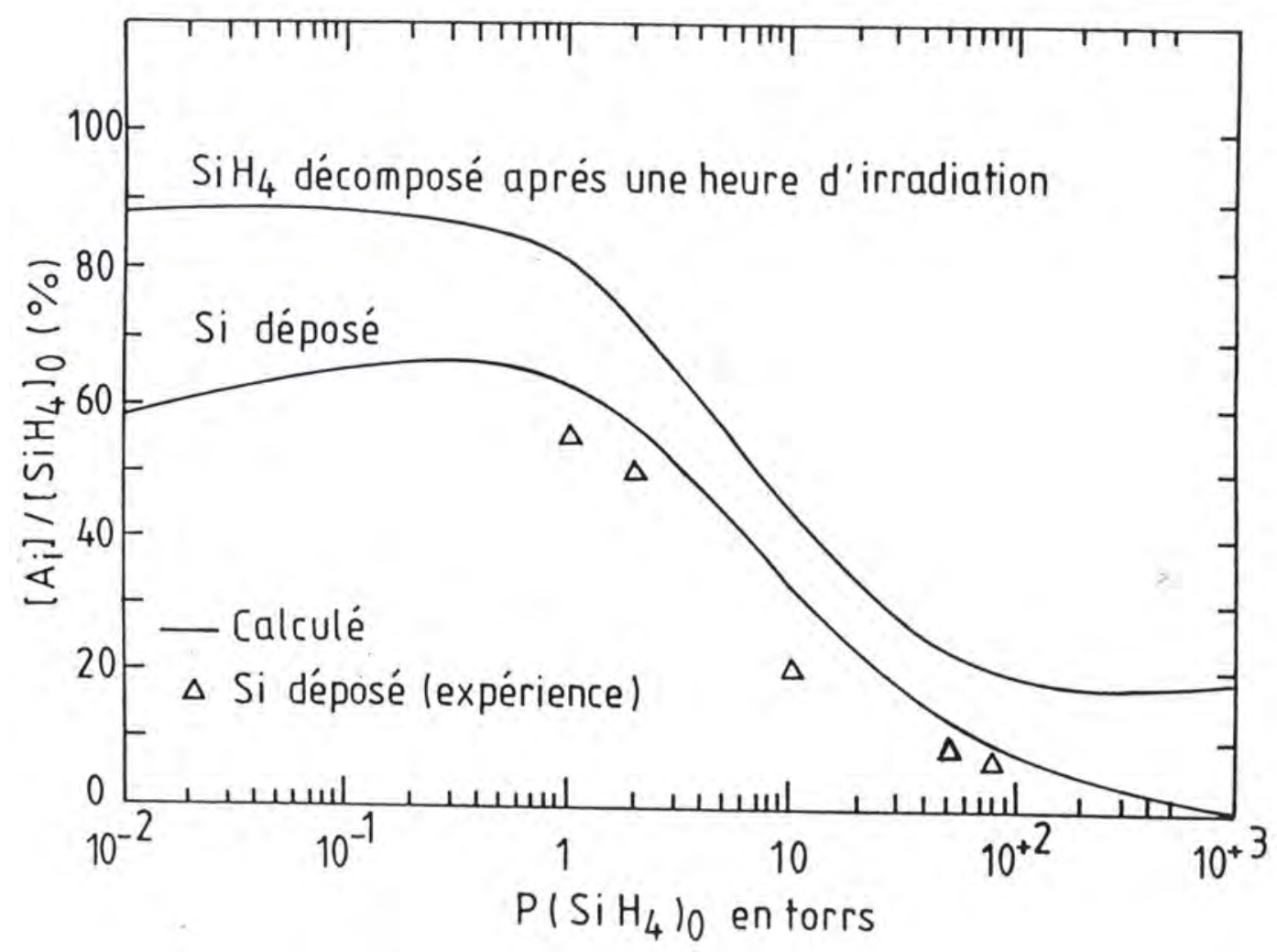

Figure 3 : Simulation de la quantité de silane décomposé et de silicium déposé en fonction de la pression initiale de silane

En dessous du Torr, la quantité relative de silane décomposé $\left(\mathrm{SiH}_{4}\right)$ décomposé / $\left(\mathrm{SiH}_{4}\right)$ initial et de silicium déposé ( $\mathrm{Si}$ / / $\left(\mathrm{SiH}_{4}\right)$ initial est très élevée, pouvant atteindre 90 et $70 \%$ respectivement de 20 à $10 \%$ à 50 Torrs. 
En vue d'obtenir le maximum de conversion de silane et d'augmenter la vitesse de dépôt, il est nécessaire d'opérer à basse pression, à forte intensité lumineuse incidente et porter le mercure liquide à une température suffisante.

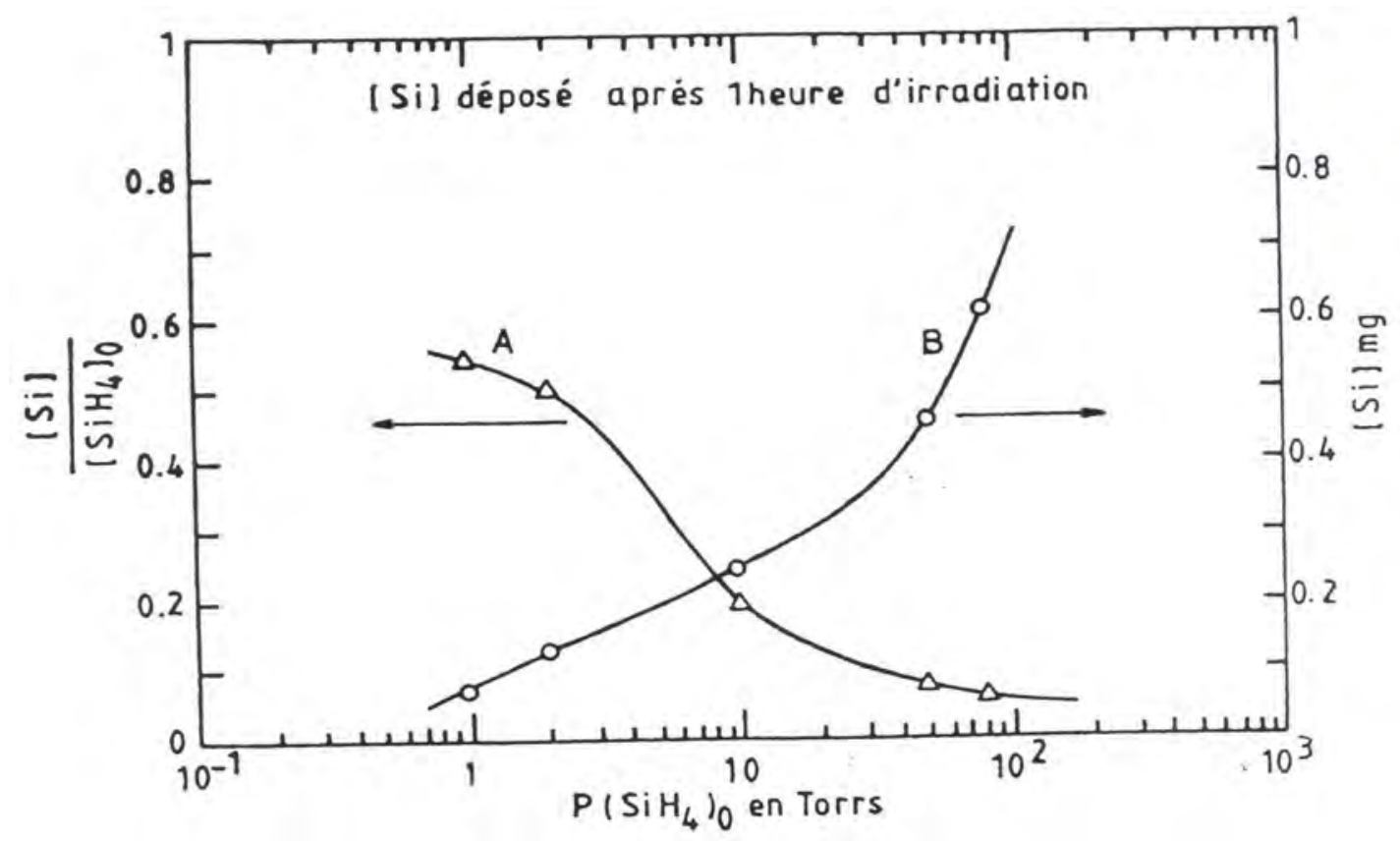

Figure 4 : Quantité de silicium déposé pendant une heure d'irradiation, en fonction de la pression initiale de silane

L'étude préalable décrite ci-dessus a été mise à profit dans la technique Hg-photo-CVD du silane pour déposer des couches minces de silicium amorphe hydrogéné a-Si:H. Les résultats publiés sur a-Si:H montrent une dispersion importante dépendant des procédés et des conditions de préparation. Les couches obtenues à partir du silane contiennent une certaine quantité d'hydrogène à la différence de celles préparées par sputtering.

Le désordre est responsable de nombreuses liaisons Si-Si brisées, se traduisent par l'apparition dans la bande interdite d'une densité importante d'états électroniques localisés, se comportant comme des pièges pour les électrons. La présence d'hydrogène a pour effet de modifier les états situés dans le gap et influencer ainsi les paramètres optiques et électriques du matériau. 
L'analyse IR a montré que les types de liaisons existantes dans le matériau sont SiH, $\mathrm{SiH}_{2}$, $\left(\mathrm{SiH}_{2}\right) \mathrm{n}$ et $\mathrm{SiH}_{3}$ dont les proportions dépendent de la température du substrat. $\mathrm{A}$ basse température, on observe les modes de vibration dûs à toutes les liaisons, s'amenuisant au fur et à mesure que la température du substrat augmente. L'analyse de la composition a montré que le rapport $(\mathrm{H} / \mathrm{Si}$ ) va de 10 à $40 \%$ quand on passe respectivement d'une température de substrat de 400 à $100^{\circ} \mathrm{C}$.

La caractérisation optique du matériau a consisté à mesurer la transmission et la réflexion des échantillons déposés sur du verre corning dans le domaine de 0,3 à 2,5 $\mu \mathrm{m}$. De ces deux mesures, les paramètres optiques, en terme d'indice de réfraction $\mathrm{n}$, de coefficient d'extinction $k$ et de coefficient d'absorption $\alpha(\alpha=(4 \pi \mathrm{k} / \lambda)$ en ont été déduites $[8,13]$.

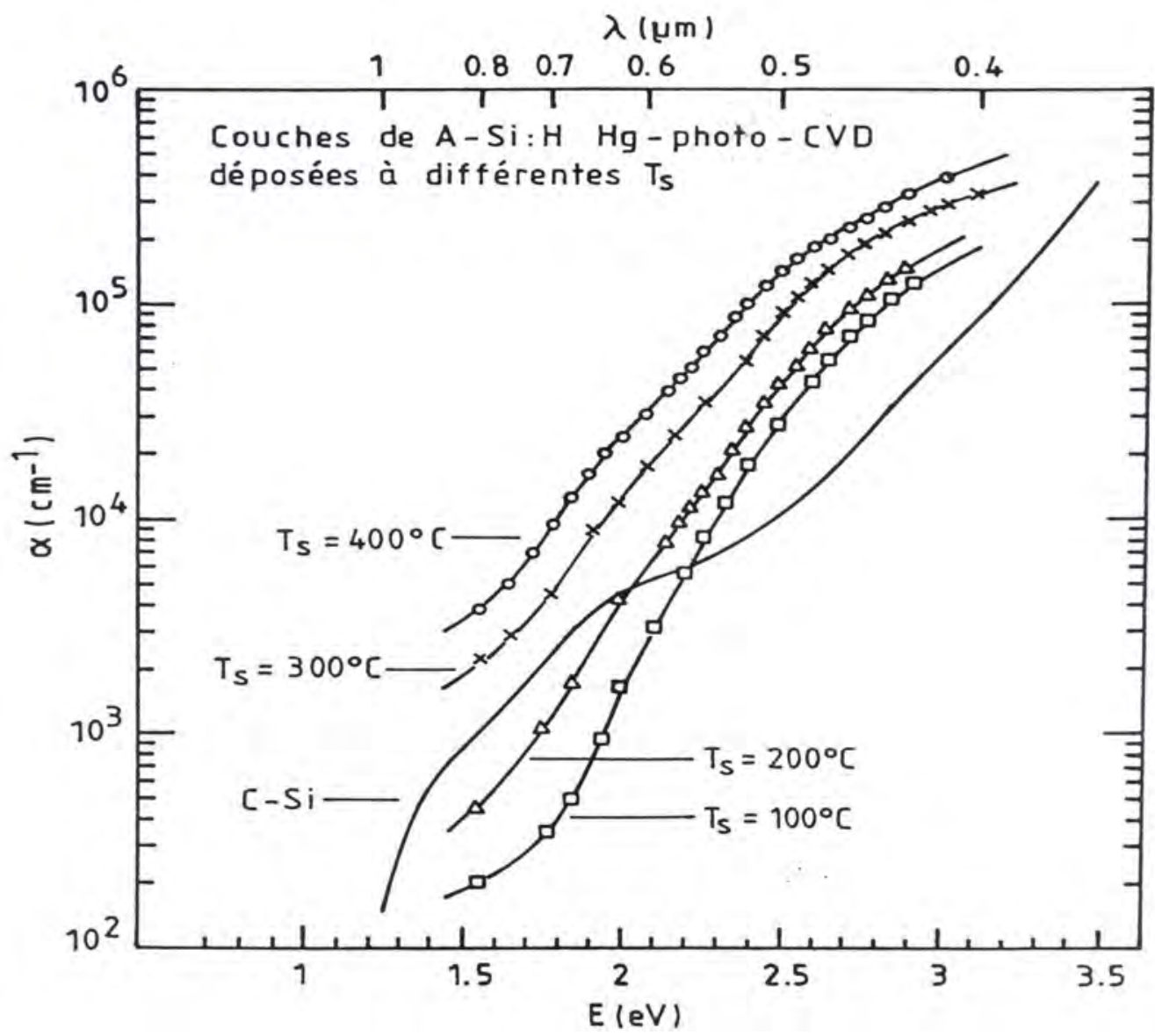

Figure 5 : Spectre d'absorption comparé entre le silicium cristallin et les couches a-Si:H 
L'épaisseur des échantillons $(<5000 \AA$ A ) a été déterminée d'une part à partir des franges d'interférences du spectre de transmission dans la zone de faible absorption $(\lambda>1 \mu \mathrm{m})$ et, d'autre part, par ellipsométrie.

Le coefficient d'absorption de nos couches dans le domaine du visible est 5 à 10 fois plus élévé que celui du silicium cristallin (Figure 5).

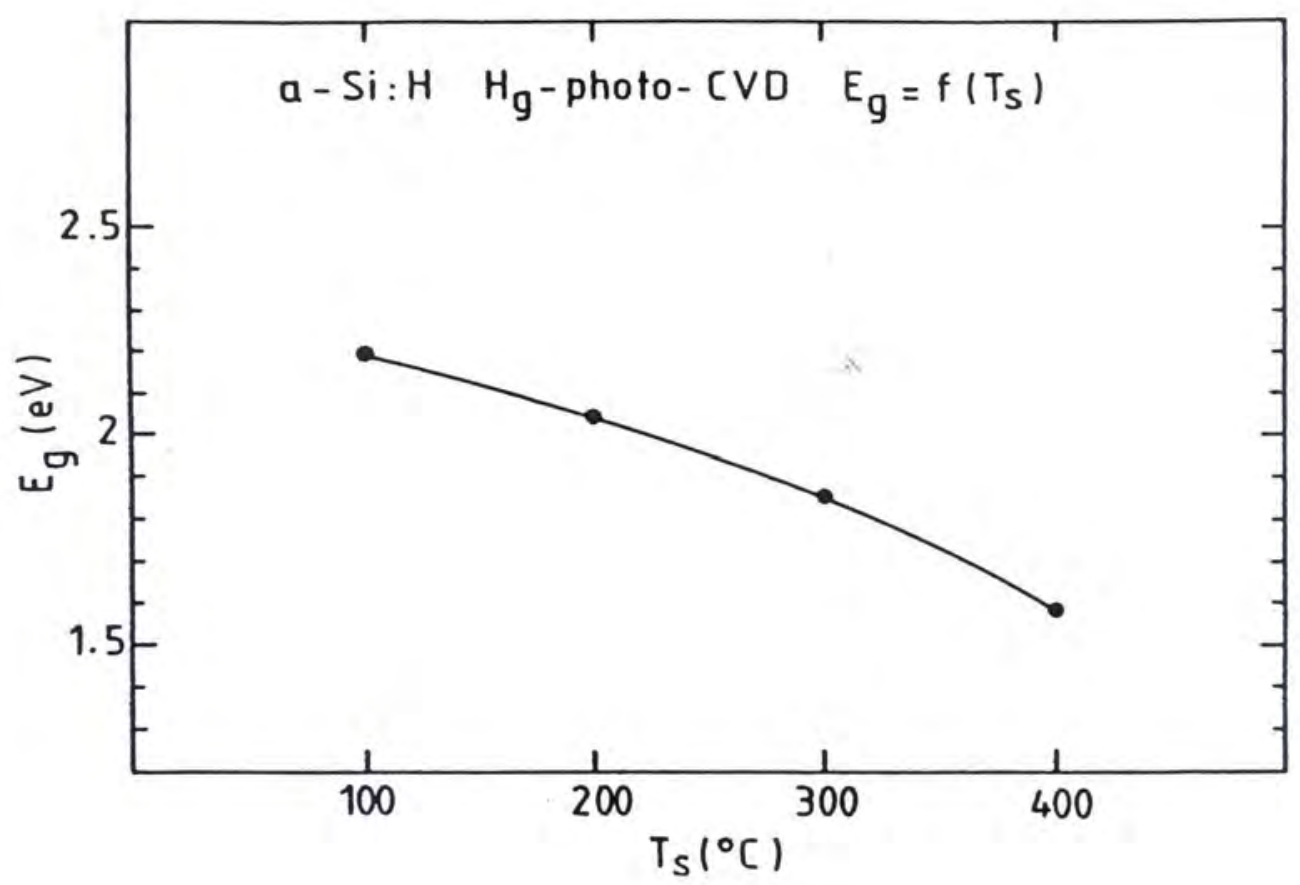

Figure 6 : Evolution du gap optique avec la température du substrat

L'étude de l'effet du recuit avait montré que le recuit des couches minces de a-Si :H, à une température supérieure à la température de dépôt, provoque un réarrangement de la matrice et un départ d'hydrogène se traduisant par une modification de leurs propriétés optiques. II serait judicieux d'opérer des recuits II doux II dans la gamme de température $200-300^{\circ} \mathrm{C}$ pendant 30 minutes.

On a observé que le coefficient d'absorption crô̂t quand on augmente soit le temps, soit la température de recuit, ayant pour conséquence une diminution du gap optique. Ceci se traduit par une réorganisation chimique et structurale du réseau, associée à une déshydrogénation partielle de la couche. Le gap optique Eg étant étroitement lié à la teneur en hydrogène de la couche. 


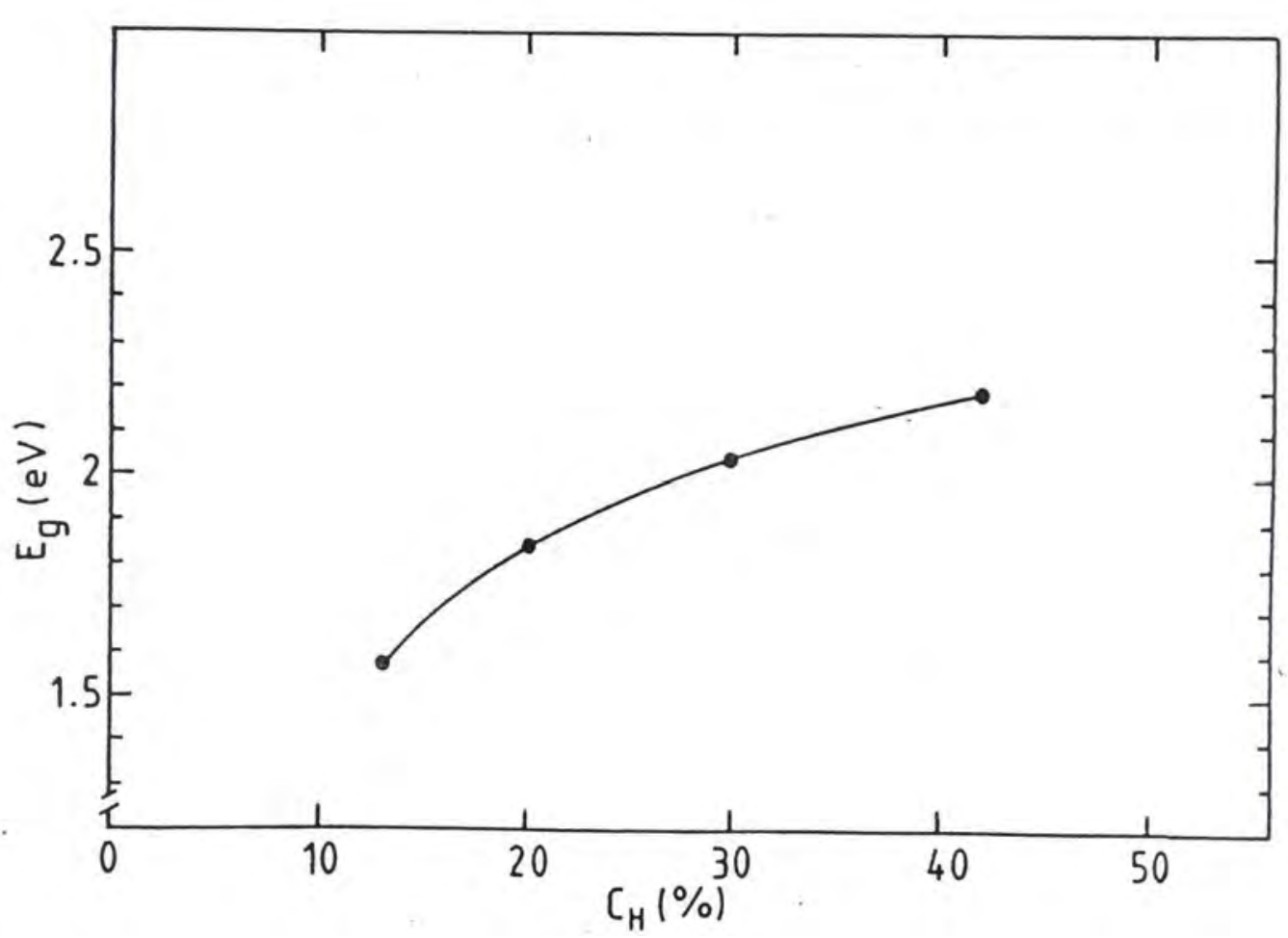

Figure 7 : Evolution du gap optique avec la teneur en hydrogène

L'étude électronique a porté sur la mesure de la conductivité et de la photoconductivité de nos échantillons en utilisant une structure coplanaire d'électrodes d'aluminium évaporées sur le film déposé sur un substrat très resistif (verre corning). Les résultats montrent un effet photosensible notable dans nos échantillons, atteignant un rapport maximum de l'ordre de $10^{3}$ sous éclairement AMI, à Ts $\sim 280^{\circ}$ C (Figure 8 et 9). 


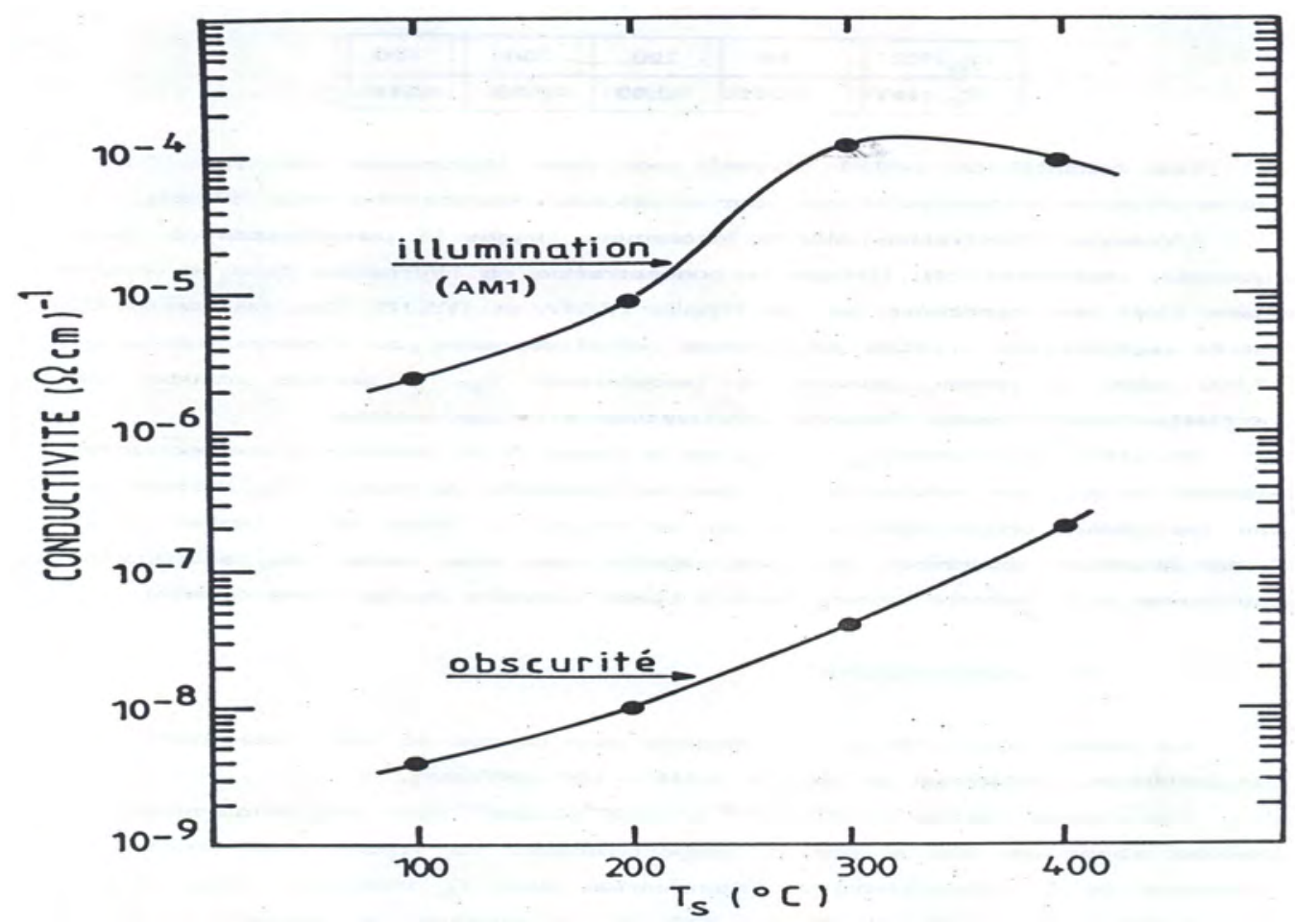

Figure 8 : Valeurs mesurées de la conductivité dans l'obscurité et sous éclairement

En outre la réponse spectrale que nous avions mesurée présente un maximum de sensibilité vers le vert $(\lambda=0,55 \mu \mathrm{m})$.

L'étude réalisée sur la dégradation des couches a-Si:H intrinsèques en fonction de la durée de l'éclairement (effet Staebler Wronski) montre que la chute de la photoconductivité est comparable à celle observée dans les autres techniques (glow discharge par exemple). 


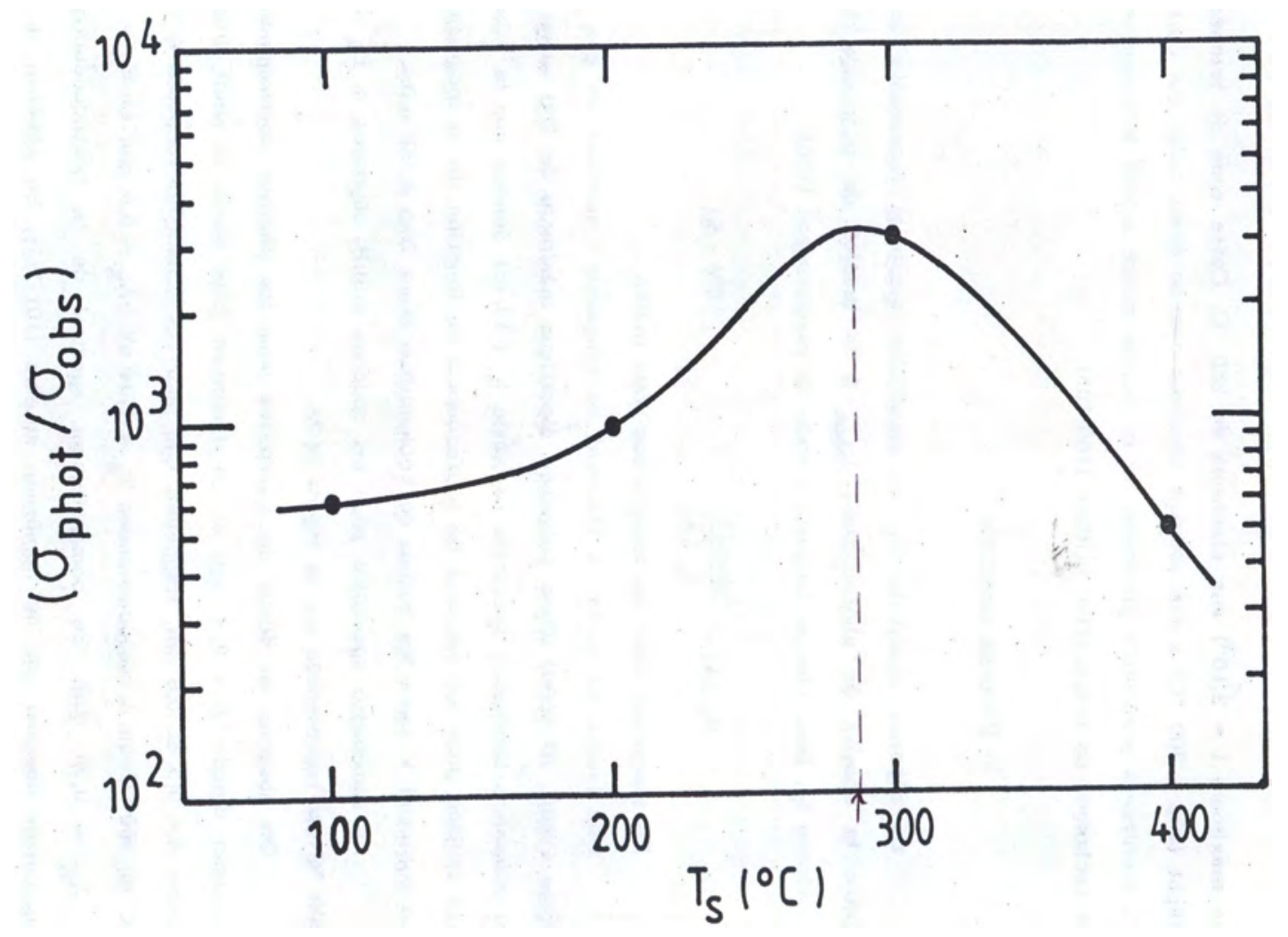

Figure 9 : Profil de la photosensibilté sous éclairement AMI

\section{Conclusion}

Les différentes mesures et simulations réalisées dans ce travail montrent que la méthode $\mathrm{Hg}$-photo-CVD est une concurrente favorisée pour le dépôt en couches minces du silicium amorphe. De meilleurs rendements de conversion de $\mathrm{SiH}_{4}$ aux basses pressions relatives notamment aux alentours de 1 Torr sont observés, en opérant notamment à une température de mercure avoisinant $50^{\circ} \mathrm{C}$.

Ainsi, pour cette technique plusieurs expériences indiquent également que les couches présentent les meilleures propriétés physico-chimiques dans l'intervalle des températures de substrat $200-300^{\circ} \mathrm{C}$.

Aujourd'hui, de nouvelles perspectives sont à entrevoir avec la méthode $\mathrm{Hg}$-photoCVD pour l'élaboration de structures nanocristallines. 


\section{Références}

[1] - H. NIKI and G. MAINS, J. Phys. Chem. 68 (2) (1964) 304

[2] - T. SAITOH, S.MURAMATSU, T. SHIMIDA et M. MIGITAKA, Appl. Phys. Lett. 42 (1983) 678

[3] - B. AKa, E. FOgarasSY, C. FUCHS. A. SLAOUI et P. SIFFERT, "Processus chimiques intervenant lors du dépôt de a-Si:H par Photo-CVD ", Actes du Colloque International sur la Science des Matériaux pour l'Energie, patronné par l'UNESCO (26 août -11 septembre) 1986 , Trieste (Italie )

[4] - B. AKA et A. TROKOUREY, "Mesure des pressions partielles des constituants gazeux résultant de la décomposition de $\mathrm{SiH}_{4}$ ou $\mathrm{Si}_{2} \mathrm{H}_{6}{ }^{\prime}$, Journal de la Société Ouest Africaine de Chimie (SOACHIM), 06 BP 9006 Ouagadougou (Burkina Faso); J. Soc. Ouest Afr. Chim. 009 (2000) $27-41$

[5] - B. AKA, G.A. MONNEHAN, C. FUCHS and E. FOGARASSY, " A comparative study of the process induced during Hg-photo-CVD and laser ArF CVD of a-Si:H films"

Revue Ivoirienne des Sciences et Technologie, Rev. Ivoir. Sci. Technol., 02 (2001) $49-56$

[6] - A. BARDHADI, Afrique Science, Vol.1(1) (2005)1-23, http://www.afriquescience.info

[7] - B. AKA, "Propriétés structurales et opto-électroniques de couches minces de silicium amorphe hydrogéné (a-Si: H) déposées par la méthode Hg-photo-CVD", J. Soc. Ouest Afr. Chim. 010 (2001) 119-139

[8] - B. AKA, " Méthode photométrique et digitale de détermination de l'épaisseur et des constantes optiques d'une couche mince absorbante", Phys. Chem. News, 001 (2001) $47-55$

[9] - B. AKA, E. BOCH, "Comparison of the processes induced by nitrogen dilution on the photodissociation of silane and disilane at $193 \mathrm{~nm}$ ", Journal of Photochemistry and Photobiology A : Chemistry, Printed by ELSEVIER science, United Kingdom (UK), J. Photochem. Photobiol. A : Chemistry, Vol I50 (2002) 257-265

[10] - R. N. KRE, M. L. MOUSSE, B. AKA et P. A. THOMAS, II Détermination des paramètres optiques du silicium amorphe évaporé pur II, Rev. Ivoir. Sci. Technol., 10 (2007) 57 -69 , Site web: http://revist.ci

[11] - B. AKA, A. BENMAKHLOUF, M. CHAFIK EL IDRISSI et A. CHOUIYAK, I Comparaison des techniques Sputtering et Hg-photo-CVD, en relation avec les propriétés optiques des dépôts a-Si:H II, Rev. Ivoir. Sci. Technol., 08 (2006) 49 - 60, Site web: http://revist.ci

[12] - A. BENMAKHLOUF, B. AKA et M. CHAFIK El IDRISSI, "I Effets du recuit thermique sur les propriétés opto-électroniques du silicium amorphe hydrogéné déposé par la technique Hg-photo-CVD II, Revue Ivoirienne des Sciences et Technologie, Rev. Ivoir. Sci. Technol, 06 (2005) 79-92, Site web : http://revist.ci 
[13] - A. BARDHADI, S. KARBAL, N. M'GAFAD and B. AKA, II Optical characterization of aSi:H thin films grown by Hg-photo-CVD II, Moroccan Journal of Condensed Matter, Rabat, Maroc, M. J. Condensed Matter, Vol. II, Nol (2009)I-8, site Web : http://www.fsr.ac.ma/MJCM/

[14] - B. AKA, C. FUCHS. E. FOGARASSY and P. SIFFERT, Evidence of a photostationary state during the mercury photosensitized decomposition of $\mathrm{SiH}_{4}$, E-MRS (EuropeanMaterials Research Society) proceedings, Strasbourg (France), Vol.XV (june 1987) 147-152, Les Editions de physique, PARIS.

[15] - B. AKA et P. SIFFERT, -"Caractérisation structurale et optique du silicium amorphe hydrogéné déposé par CVD laser ArF de Si2H6", J. Soc. Ouest Afr. Chim. 012 (2001) $63-80$

[16] - C. FUCHS, E. BOCH, E. FOGARASSY, B. AKA and P. SIFFERT, "Two-photon absorption cross section of silane under pulsed ArF (193 nm) excimer laser irradiation", MRS (Materials Research Society) proceedings, Boston (USA), Vol.101 (1988) 361-365 